\title{
IMPACTO DE LA CIRUGÍA DE CATARATA: AGUDEZA VISUAL Y CALIDAD DE VIDA
}

\section{IMPACT OF CATARACT SURGERY ON VISUAL ACUITY AND QUALITY OF LIFE}

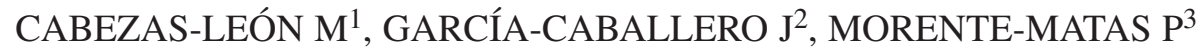

\section{RESUMEN}

Objetivo: Medir el impacto de la cirugía de catarata sobre la calidad de vida y agudeza visual.

Métodos: Se mide la calidad de vida en pacientes intervenidos de Catarata de cuarenta y seis a noventa y dos años de edad, de ambos géneros y con un nivel intelectual que les permitiera comprender el cuestionario (SF-36). Se completó el cuestionario antes del acto quirúrgico seis y dieciocho meses después. La agudeza visual se mide con el optotipo de Snellen.

Resultados: Se realizaron 150 cuestionarios. La edad media fue de 74,32 años (DE 6,44). El dolor corporal fue mejorando de forma significativa. La función social, salud general, evolución declarada de la salud, rol físico y función física tienen una evolución similar, manteniendo una mejoría significativa entre la primera y tercera consulta. No existieron diferencias significativas en el rol emocional ni en la vitalidad. La salud mental ha ido empeorando en las sucesivas consultas con diferencias significativas entre ellas. Los valores medios de agudeza visual son $18,39 \%, 66,01 \%$ y $69,02 \%$.

Conclusiones: La facoemulsificación es un método efectivo y seguro para mejorar la calidad de vida, sobre todo en los aspectos físicos. La disminución

\section{ABSTRACT}

Objective: To evaluate visual acuity and quality of life improvement after cataract surgery.

Methods: Visual acuity and quality of life were assessed in patients undergoing phaco-emulsification. They were of both genders, ranged from forty-six to ninety-two years of age, and were able to understand the SF-36 quality of life questionnaire which explores the differences in quality of life before and after surgery. The questionnaire was completed before cataract surgery and again 6 and 18 months later. Visual acuity was measured with Snellen optotype.

Results: The answers of 150 patients were evaluated in this study. The average age was 74.32 years. After the surgery the body pain results increased, with this shown to be statistically significant. The social function, general health, perception, role limitation due to physical problems and physical function significantly improved between the first and third consultations. There were no significant differences in role limitation due to emotional problems, nor in vitality. The mental health dimension became worse between consultations although there was no statistically significant difference found. The average values for Visual Acuity were 18.39, 66.01 and 69.02 at the three timed assessments made.

\footnotetext{
Recibido: 9/4/07. Aceptado: 6/3/08.

1 Doctora en Medicina. Especialista en Oftalmología. Hospital Virgen de la Salud. Toledo. España.

2 Doctor en Medicina. Especialista en Medicina Preventiva. Hospital Universitario La Paz. Madrid. España.

${ }^{3}$ Licenciada en Medicina. Especialista en Oftalmología. Hospital Virgen de la Salud. Toledo. España.

Los autores certifican que resultados parciales de este trabajo han sido publicados en:

- Comunicación en Panel: «Calidad de vida en pacientes intervenidos de catarata». VI Jornadas Nacionales sobre avances en Medicina Preventiva. Archa, junio 2004.

- Artículo original: «Calidad de vida en pacientes intervenidos de catarata». Archivos de la Sociedad Española de Oftalmología, agosto 2005.
}

Correspondencia:

Mercedes Cabezas León

C/. Potosí, 2

45004 Toledo

España

E-mail: mercedescabezasleon@yahoo.es 
de la limitación de roles debido a problemas de salud físicos tras la cirugía es concordante con la mejora física.

La agudeza visual aumenta claramente tras la cirugía. Los cuestionarios genéricos de calidad de vida como el SF-36 se pueden utilizar como medida de resultado de la cirugía de catarata.

Palabras clave: Catarata, agudeza visual, calidad de vida, Cuestionario SF-36.

\section{INTRODUCCIÓN}

La prevalencia de cataratas seniles en la población general se incrementa hasta el 45,9\% entre los 75 y 85 años $(1,2)$. En muchos países desarrollados la cirugía de catarata es uno de los procedimientos quirúrgicos más realizados en la población adulta (3).

Lo que se define como calidad de vida en relación a la salud tiene dos partes, que presentan diferentes problemas a la hora de validarse $(4,5)$ : la parte objetiva que es el estatus funcional del individuo y la parte subjetiva que es el sentimiento de salud, la sensación de bienestar (6). Incluye:

Funcionalidad física: posibilidad de llevar a cabo actos de la vida diaria como higiene y cuidado personal, pasear... La dimensión física de la calidad de vida también hace referencia a los síntomas de enfermedad y sus tratamientos.

Funcionalidad psicológica: bienestar mental y emocional, satisfacción y felicidad.

Funcionalidad social: relaciones sociales, participación en actividades.

Percepción de la salud, del dolor y sobre todo satisfacción sobre la propia vida.

En los últimos años se ha impulsado el estudio de la valoración de la función visual y de la calidad de vida relacionada con la visión. Este aumento de interés ha llevado al desarrollo de instrumentos, en forma de cuestionarios, que intentan medir estos conceptos. Si pudiésemos medir de forma cuantitativa la afectación de la calidad de vida producida por la catarata tendríamos un parámetro objetivo para valorar su progresión, la indicación de la cirugía y la mejoría tras ella. La finalidad de la cirugía de catarata es mejorar la agudeza visual y por tanto
Conclusions: Cataract surgery performed by phacoemulsification has been proven to be effective in improving quality of life, especially in physical aspects, according to the improvement in role limitation due to physical problems. Visual acuity also improves after surgery. Disease specific instruments, such as the SF-36, should be used as the outcome measure in clinical practice after cataract surgery (Arch Soc Esp Oftalmol 2008; 83: 237-248).

Key words: Cataract, Visual acuity, Quality of life, SF-36 Survey.

la función visual considerando que esto implica una mejoría en la calidad de vida. En el año 2005 se ha publicado un estudio observacional hecho por Lee y colaboradores, cuyos resultados indicaron que los síntomas de la catarata están altamente asociados con la calidad de vida relacionada con la visión (7).

Se decidió emplear el cuestionario SF36 por ser genérico, multidimensional y accesible. Se pretende demostrar lo que se gana en función visual y calidad de vida (relacionada con la salud y con la visión) tras la cirugía de catarata (8).

Se parte de la hipótesis de la existencia de un impacto importante de la cirugía de catarata en la calidad de vida y en la agudeza visual de pacientes intervenidos de esta patología en la Unidad de Cirugía Mayor Ambulatoria del Servicio de Oftalmología del Hospital Virgen de la Salud (Toledo). Dicho impacto se puede cuantificar utilizando un cuestionario ya validado (SF-36) y el optotipo para visión lejana de Snellen.

Los objetivos son:

- Hacer objetivable la mejora de la agudeza visual tras la cirugía de catarata y presentar un instrumento para medir directamente la calidad de vida.

- A través de un cuestionario (SF-36) aplicado a pacientes con cataratas, medir la afectación funcional y la ganancia de la misma tras la cirugía. Valorar, en base al análisis de los resultados del SF36 , la variación en calidad de vida pre y post cirugía.

- Valorar, en base al registro de la agudeza visual según el optotipo de Snellen, la variación en la mejor visión corregida pre y postcirugía.

- Relacionar los cambios en agudeza visual y calidad de vida, pre y postcirugía. 


\section{SUJETOS, MATERIAL Y MÉTODOS}

\section{Tipo de estudio}

Estudio prospectivo y analítico longitudinal, de base hospitalaria, de la medición de la calidad de vida con el cuestionario SF-36 y de la mejor agudeza visual corregida con el optotipo para visión lejana de Snellen, en pacientes intervenidos de catarata en la Unidad de Cirugía Mayor Ambulatoria del Servicio de Oftalmología del Hospital Virgen de la Salud de Toledo.

\section{Población de estudio}

Conjunto de 150 pacientes intervenidos de Catarata del primer ojo mediante facoemulsificación con implante de lente intraocular, entre los meses de octubre de 2002 y junio de 2003.

\section{Criterios de inclusión}

Pacientes a los que se les haya realizado facoemulsificación de cristalino con posterior colocación de lente intraocular, de cuarenta y seis a noventa y dos años de edad, de ambos géneros y con un nivel intelectual que les permita comprender y realizar el cuestionario. Incluyendo pacientes con patología oftalmológica previa, como glaucoma o degeneración macular, y sin descartar a los que presentaron complicaciones intra o postoperatorias.

\section{Estudio de campo}

\section{Cuestionario SF-36 (Anexo 1)}

Se utiliza como método de exploración para medir la evaluación subjetiva de los resultados de la cirugía de catarata por parte de los pacientes (9-11). El cuestionario SF-36 (Short-Form-36 Health Survey) fue elaborado por el Dr. John Ware a partir del MOS (Estudio de resultados médicos del grupo Rand). Es un instrumento de medida del estado de salud general y de resultados de intervenciones médicas. Puede ser auto-administrado ó completado por un entrevistador. Se decide emplear este cuestionario por ser uno de los métodos de valoración subjetiva más utilizado tras la cirugía de cata- rata. El cuestionario se completó antes del acto quirúrgico, seis meses y dieciocho meses después.

\section{Optotipo de Snellen}

Es un optotipo de medición de visión lejana.

- Variables independientes:

Género.

Edad: en años.

Fechacx: fecha de la cirugía.

Las siguientes variables se recogieron en tres ocasiones: una en la consulta previa a la cirugía, otra a los seis meses de la cirugía y una tercera a los dieciocho meses de la misma, representándose con el subíndice 1, 2 y 3 respectivamente:

Fecha de realización del cuestionario SF-36.

Medición de la agudeza visual.

Variables correspondientes al cuestionario de calidad de vida SF-36: Función física, Limitación de roles debido a problemas físicos, Dolor corporal, Función social, Salud mental, Bienestar emocional, Limitación de roles por problemas emocionales, Vitalidad, energía o fatiga, Percepción de la salud general y Salud comparada con la de hace un año.

- Variable dependiente o resultado:

Calidad de Vida mejorada, igual o empeorada. Se define en base a los resultados de medición de la calidad de vida por medio del cuestionario SF-36 antes y tras la cirugía de catarata. Podemos definir:

- Calidad de vida mejorada: mejor puntuación postcirugía que precirugía.

- Calidad de vida igual: misma puntuación pre y postcirugía.

- Calidad de vida empeorada: peor puntuación postcirugía que precirugía.

Agudeza visual mejorada, igual o empeorada. Se definió de acuerdo con los resultados de registro mediante el sistema decimal de la mejor visión corregida a través del optotipo para visión lejana de Snellen. Sirve para valorar la calidad de vida relacionada con la salud.

- Circuito de recogida de datos.

El paciente acudía a la consulta y se hacía una primera valoración (anamnesis + exploración); se cumplimentaba la inclusión en lista de espera quirúrgica de catarata y realizaba el cuestionario SF36. Los cuestionarios postcirugía se realizaron mediante entrevista telefónica.

El período de asistencia establecido para incluirlo en el estudio fue de nueve meses para la recogi- 


\section{Cuestionario de Salud SF-36}

INSTRUCCIONES: Las preguntas que siguen se refieren a lo que usted piensa sobre su salud. Sus respuestas permitirán saber cómo se encuentra usted y hasta qué punto es capaz de hacer sus actividades habituales. Conteste cada pregunta tal como se indica. Si no está seguro de cómo responder a una pregunta, por favor conteste lo que le parezca más cierto.

1. En general, usted diría que su salud es: (marque un solo número)

$\begin{array}{ll}\text { Excelente } & 1 \\ \text { Muy buena } & 2 \\ \text { Buena } & 3 \\ \text { Regular } & 4 \\ \text { Mala } & 5\end{array}$

2. ¿Cómo diría usted que es su salud actual, comparada con la de hace un mes? (marque un solo número)

Mucho mejor ahora que hace un mes

Algo mejor ahora que hace un mes 2

Más o menos igual que hace un mes $\quad 3$

Algo peor ahora que hace un mes 4

Mucho peor ahora que hace un mes $\quad 5$

3. Las siguientes preguntas se refieren a actividades o cosas que usted podría hacer en un día normal. Su salud, ¿le limita para hacer esas actividades o cosas? Si es así, ¿cuánto? (marque solo un número por cada pregunta)

Actividades Sí, me limita mucho Sí, me limita un poco No, no me limita nada
a) Esfuerzos intensos, tales como correr,
levantar objetos pesados, o participar
en deportes agotadores
b) Esfuerzos moderados, como mover una mesa, pasar la aspiradora, jugar a los bolos o caminar más de 1 hora
c) Coger o llevar la bolsa de la compra
d) Subir varios pisos por la escalera
e) Subir un solo piso por la escalera
f) Agacharse o arrodillarse
g) Caminar un kilómetro o más
h) Caminar varias manzanas (varios centenares de metros)
i) Caminar una sola manzana
j) Bañarse o vestirse por sí mismo

$\begin{array}{ll}1 & 2 \\ 1 & 2 \\ & \\ 1 & \\ 1 & 2 \\ 1 & 2 \\ 1 & 2 \\ 1 & 2 \\ 1 & 2 \\ 1 & 2 \\ 1 & 2 \\ & 2\end{array}$
3
2
3

$\begin{array}{ll}2 & 3 \\ 2 & 3 \\ 2 & 3 \\ 2 & 3 \\ 2 & 3 \\ 2 & 3 \\ 2 & 3 \\ 2 & 3\end{array}$

4. Durante las últimas 4 semanas, ¿ha tenido alguno de los siguientes problemas en su trabajo o en sus actividades cotidianas, a causa de su salud física? (marque un solo número por cada pregunta)

$\begin{array}{llrr} & & \text { SÍ } & \text { NO } \\ \text { a) ¿Tuvo que reducir el tiempo dedicado al trabajo o a sus actividades cotidianas? } & 1 & 2 \\ \text { b) ¿Hizo menos de lo que hubiera querido hacer? } & 1 & 2 \\ \text { c) iTuvo que dejar de hacer algunas tareas en su trabajo o en sus actividades cotidianas? } & 1 & 2 \\ \text { d) ¿Tuvo dificultad para hacer su trabajo o sus actividades cotidianas } & 1 & 2\end{array}$

5. Durante las 4 últimas semanas, ¿ha tenido alguno de los siguientes problemas en su trabajo o en sus actividades cotidianas, a causa de algún problema emocional (como estar triste, deprimido, o nervioso? (marque un solo número por cada pregunta)

\begin{tabular}{llcc}
\hline & Sí & NO \\
\hline a) ¿Tuvo que reducir el tiempo dedicado al trabajo o a sus actividades cotidianas, & 1 & 2 \\
por algún problema emocional? & 1 & 2 \\
b) ¿Hizo menos de lo que hubiera querido hacer, por algún problema emocional? & 1 & 2 \\
c) ¿No hizo su trabajo o sus actividades cotidianas tan cuidadosamente como de costumbre, & \\
& por algún problema emocional? & \\
\hline \hline
\end{tabular}




\section{Anexo I. Cuestionario SF-36 (continuación)}

6. Durante las 4 últimas semanas, ¿hasta qué punto su salud física o los problemas emocionales han dificultado sus actividades sociales habituales con la familia, los amigos, los vecinos u otras personas? (marque un solo número)
Nada
Un poco
Regular
Bastante
1
Mucho
2
3
4
5

7. ¿Tuvo dolor en alguna parte del cuerpo durante las 4 últimas semanas?

(marque un solo número)

No, ninguno

Sí, muy poco

Sí, un poco

Sí, moderado

Sí, mucho

Sí, muchísimo

4 últimas semanas?

D. Durante las 4 últimas semanas, ¿hasta qué punto el dolor le ha dificultado su trabajo habitual (incluido el trabajo fuera de casa y las tareas domésticas)?

(marque un solo número)

Nada

Un poco

Regular

Bastante

Mucho

2

3

4

5

6

9. Las preguntas que siguen se refieren a cómo se ha sentido y cómo le han ido las cosas durante las últimas 4 semanas. En cada pregunta responda lo que se parezca más a cómo se ha sentido usted. Durante las últimas 4 semanas ¿cuánto tiempo... (marque un solo número por cada pregunta)

\begin{tabular}{|c|c|c|c|c|c|c|}
\hline & Siempre & $\begin{array}{c}\text { Casi } \\
\text { siempre }\end{array}$ & $\begin{array}{c}\text { Muchas } \\
\text { veces }\end{array}$ & $\begin{array}{l}\text { Algunas } \\
\text { veces }\end{array}$ & $\begin{array}{c}\text { Sólo } \\
\text { alguna vez }\end{array}$ & Nunca \\
\hline a) ¿Se sintió llena de vitalidad? & 1 & 2 & 3 & 4 & 5 & 6 \\
\hline b) ¿Estuvo muy nerviosa? & 1 & 2 & 3 & 4 & 5 & 6 \\
\hline c) ¿Se sintió tan baja de moral que nada podía animarle? & 1 & 2 & 3 & 4 & 5 & 6 \\
\hline d) ¿Se sintió calmada y tranquila? & 1 & 2 & 3 & 4 & 5 & 6 \\
\hline e) ¿Tuvo mucha energía? & 1 & 2 & 3 & 4 & 5 & 6 \\
\hline f) ¿Se sintió desanimada y triste? & 1 & 2 & 3 & 4 & 5 & 6 \\
\hline g) ¿Se sintió agotada? & 1 & 2 & 3 & 4 & 5 & 6 \\
\hline h) ¿Se sintió feliz? & 1 & 2 & 3 & 4 & 5 & 6 \\
\hline i) ¿Se sintió cansada? & 1 & 2 & 3 & 4 & 5 & 6 \\
\hline
\end{tabular}

10. Durante las últimas 4 semanas, ¿con qué frecuencia la salud física o los problemas emocionales le han dificultado sus actividades sociales (como visitar a los amigos o familiares? (marque un solo número)

Siempre

Casi siempre

Algunas veces

Sólo alguna vez

Nunca

1

2

3

4

5

11. Por favor, diga si le parece CIERTA o FALSA cada una de las siguientes frases:

(marque un solo número por cada pregunta)

Totalmente Bastante No lo sé Bastante Totalmente cierta cierta falsa falsa

a) Creo que me pongo enfermo más fácilmente que otras personas

b) Estoy tan sano como cualquiera

c) Creo que mi salud va a empeorar

d) Mi salud es excelente

1
1
1
1

2
2
2
2

$\begin{array}{lll}3 & 4 & 5 \\ 3 & 4 & 5 \\ 3 & 4 & 5 \\ 3 & 4 & 5\end{array}$


da de cuestionarios preintervención (octubre 2002junio 2003) y de diez meses para el postintervención (marzo-diciembre 2003) y otros nueve meses para el último registro (abril-diciembre 2004).

- Análisis de los datos:

- ESTADÍSTICA DESCRIPTIVA: Las variables cuantitativas se expresan con media \pm desviación típica, mediana, mínimo y máximo. Las variables cualitativas se expresarán por medio de frecuencias absolutas y relativas (o porcentajes).

- ESTADÍSTICA ANALÍTICA: Se aplicaron tests de Friedman para conocer si existían diferencias entre los valores obtenidos en las distintas consultas para cada una de las dimensiones de la calidad de vida y para los valores de agudeza visual. Se aplicaron posteriormente los tests post hoc correspondientes para ver en aquellos casos en los que existen diferencias entre cual de las consultas estás son significativas y un modelo lineal generalizado para valorar la tendencia lineal. El procesamiento y análisis informático de los datos se realizaron con la ayuda del paquete estadístico SPSS vs 9.0. (SPSS Inc, Illinois, USA) Se considera estadísticamente significativo un valor bilateral de $\mathrm{p}$ inferior a 0,05 .

\section{RESULTADOS}

\section{Variables de filiación}

- Género: La variable género está definida para los 150 pacientes intervenidos incluidos en la base, 70 hombres $(46,7 \%)$ y 80 mujeres $(53,3 \%)$.

- Edad: Se conoce la variable edad de los 150 pacientes intervenidos, el paciente más joven tenía 46 años, el más anciano 92 años, la media de edad es de 74,32 años, la desviación estándar de 6,44 y la mediana de 75,00 años.

\section{Variables de tiempo}

Se conoce el tiempo transcurrido desde la consulta prequirúrgica hasta el día de la cirugía en 148 pacientes. Esta media de tiempo fue de 20,14 días. La mediana fue de 14,00 días. Se conoce el tiempo transcurrido desde el día de la cirugía hasta la primera consulta postquirúrgica en 149 pacientes. La media de tiempo fue de175,78 días (5,75 meses). La mediana fue 175,00 días (5,73 meses). La media del tiempo transcurrido desde la consulta prequirúrgica hasta la segunda consulta postquirúrgica ha sido de 540,00 días $(17,67$ meses)y la mediana de 536,31 días. (17,55 meses).

\section{Cuestionario SF-36}

Los valores obtenidos para cada uno de las dimensiones de la calidad de vida se muestran a continuación.

1. Función física (fig. 1).

La función física, mejora significativamente entre la primera y segunda consulta y empeora significativamente entre la segunda y la tercera consulta, manteniendo no obstante una mejoría significativa entre la primera y tercera consulta.

2. Limitación de roles debido a problemas de salud físicos (fig. 2).

Las limitaciones de rol por problemas físicos, según se interpreta en el SF-36, mejoran tras la cirugía, ya que cuanta más puntuación mejor estado de salud según el cuestionario, y de modo estadísticamente significativo. Este aumento también aparece a los dieciocho meses respecto a la puntuación precirugía, aunque no tan llamativo como a los seis meses. La limitación de roles debido a problemas de salud físicos mejora significativamente entre la primera y segunda consulta y empeora significativamente entre la segunda y la tercera consulta, manteniendo no obstante una mejoría significativa entre la primera y tercera consulta.

3. Dolor corporal.

Función física

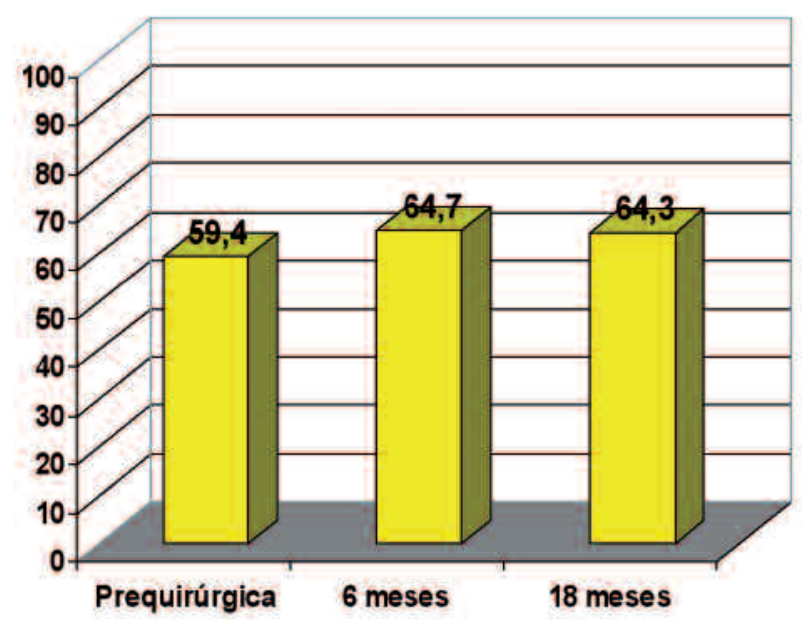

Fig. 1: Función física. 


\section{Rol físico}

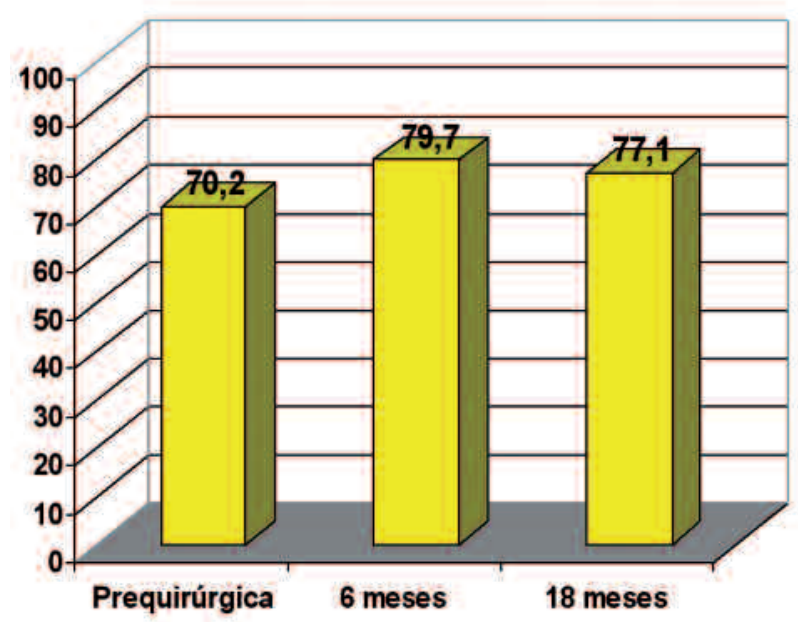

Fig. 2: Limitación de roles debido a problemas de salud físicos.

El dolor corporal ha ido mejorando en las sucesivas consultas y hay diferencias significativas entre todas ellas (fig. 3).

4. Función social: La función social mejora significativamente entre la primera y segunda consulta y mejora aunque no significativamente entre la segunda y la tercera, manteniendo no obstante una mejoría significativa entre la primera y tercera consulta (fig. 4).

5. Salud mental, bienestar emocional: La dimensión salud mental ha ido empeorando en las sucesi-

\section{Dolor corporal}

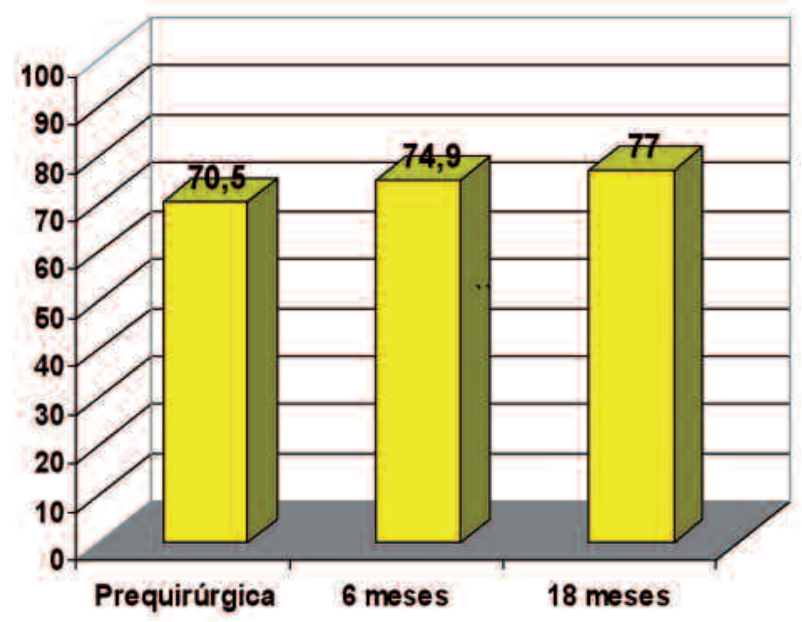

Fig. 3: Dolor corporal.

\section{Función social}

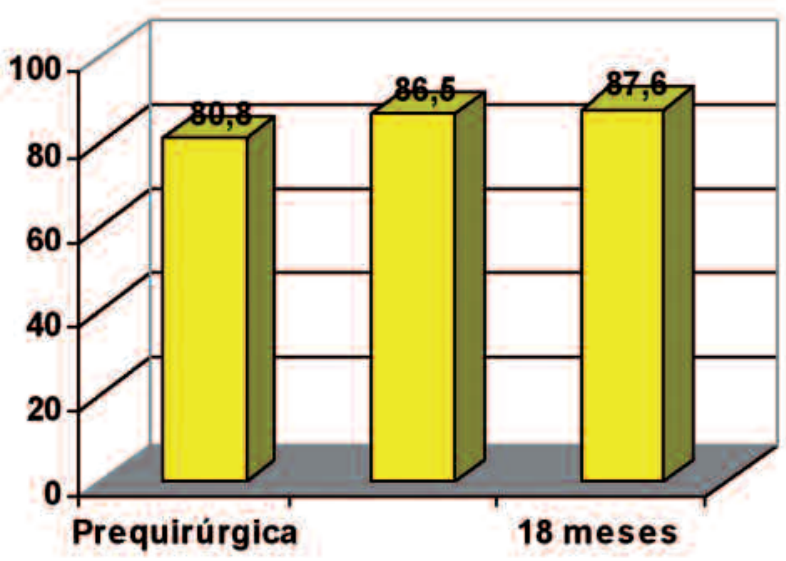

Fig. 4: Función social.

vas consultas y hay diferencias significativas entre todas ellas (fig. 5).

6. Limitación de roles debido a problemas emocionales: No existieron diferencias significativas (fig. 6).

7. Vitalidad, energía ó fatiga: No existieron diferencias significativas (fig. 7).

8. Percepción de la salud general: La percepción de la salud general mejora significativamente entre la primera y segunda consulta y empeora significativamente entre la segunda y la tercera consulta, manteniendo no obstante una mejoría significativa entre la primera y tercera consulta (fig. 8).

9. Salud comparada con la de hace un mes: La evolución declarada de la salud mejora significati-

\section{Salud mental}

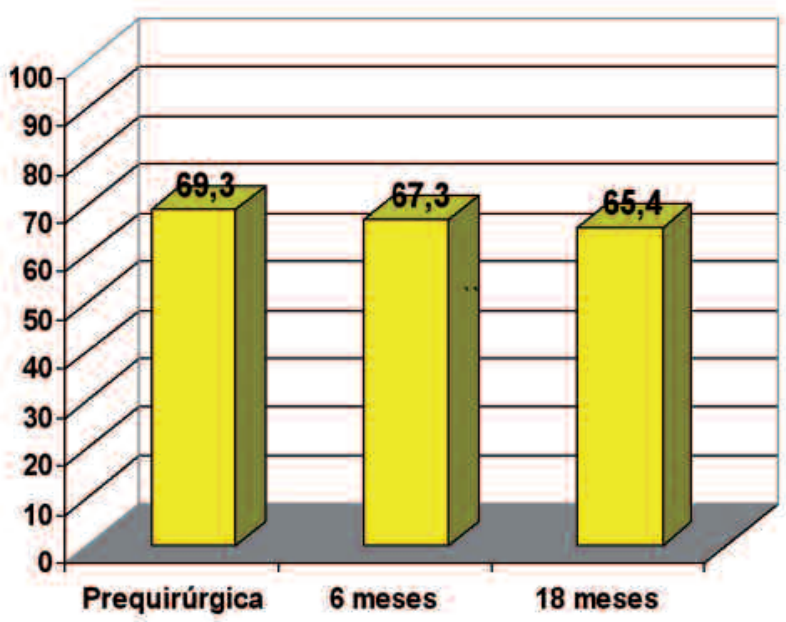

Fig. 5: Salud mental. 


\section{Rol emocional}

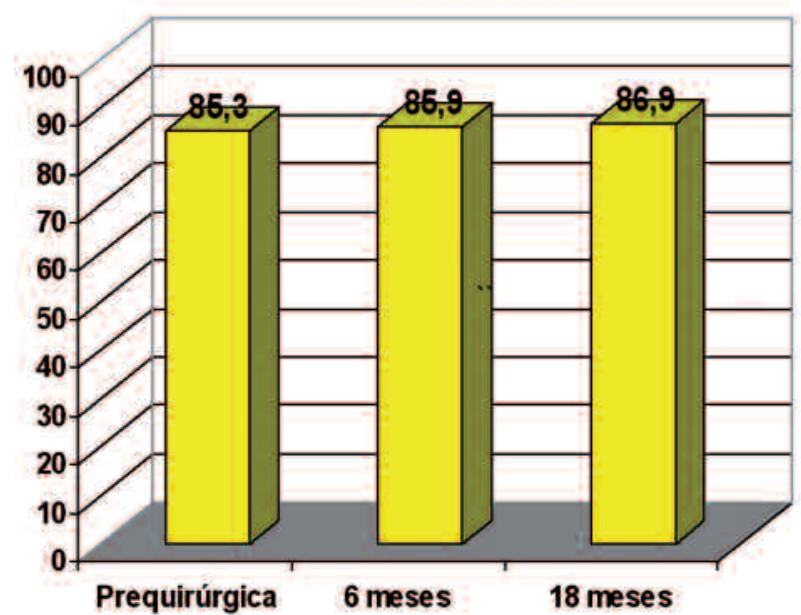

Fig. 6: Limitación de roles debido a problemas emocionales.

vamente entre la primera y segunda consulta y empeora significativamente entre la segunda y la tercera consulta, manteniendo una mejoría significativa entre la primera y tercera consulta (fig. 9).

De las nueve dimensiones del SF-36, el dolor corporal ha ido mejorando en las sucesivas consultas y hay diferencias significativas entre todas ellas. La función social, salud general, evolución declarada de la salud, rol físico y función física tienen una evolución similar, mejoran significativamente entre la primera y segunda consulta y empeoran significativamente entre la segunda y la tercera consulta, manteniendo no obstante una mejoría significativa

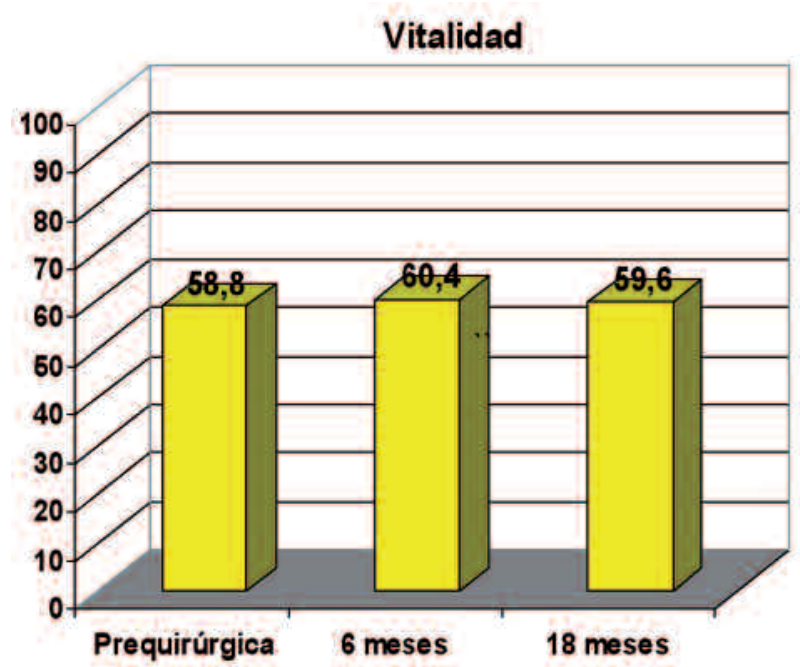

Fig. 7: Vitalidad, energía o fatiga.

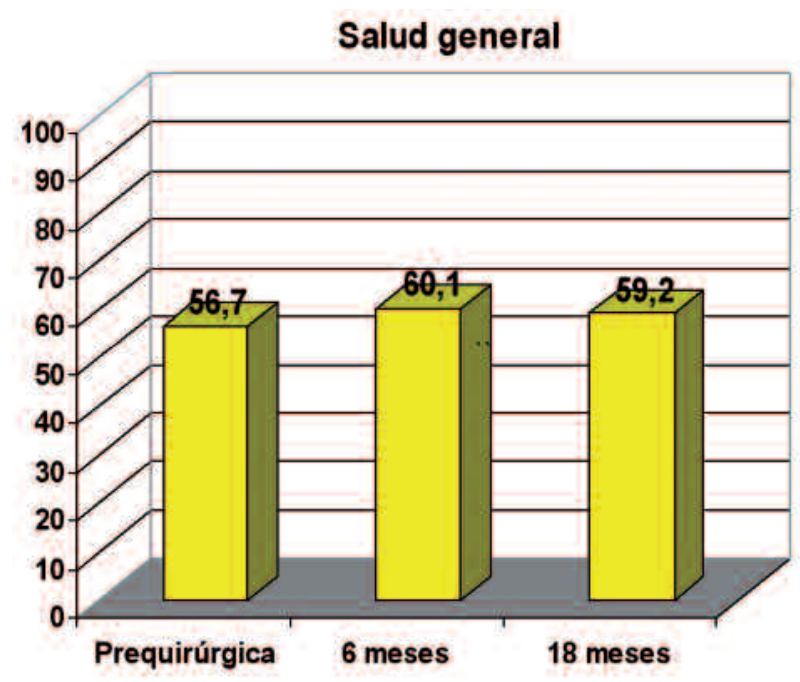

Fig. 8: Percepción de la salud general.

entre la primera y tercera consulta. No existieron diferencias significativas en el rol emocional ni en la vitalidad. La dimensión salud mental ha ido empeorando en las sucesivas consultas y hay diferencias significativas entre todas ellas.

Se realizó un modelo lineal generalizado que demuestra que en las dimensiones de la calidad de vida en las que existe una tendencia lineal (todas excepto el rol emocional y la vitalidad) entre las distintas visitas esta es independiente del sexo y de la edad de los pacientes.

Agudeza visual. Los valores medios de agudeza visual son del 18,39, 66,01 y 69,02 para las tres consultas respectivamente.

\section{Evolución declarada de la salud}

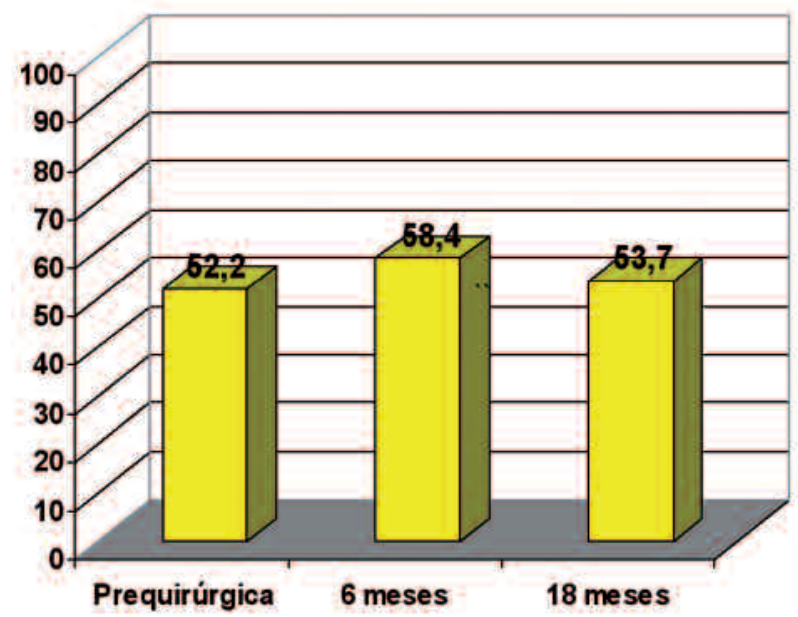

Fig. 9: Salud comparada con la de hace un mes. 
Se aplicó el test de Friedman y los test post hoc correspondientes para conocer si existían diferencias entre los valores obtenidos en las distintas consultas y se comprobó que efectivamente la agudeza visual aumentó de forma estadísticamente significativa entre todas ellas. Se realiza por otro lado un modelo lineal generalizado que demuestra que existe una tendencia lineal hacia el aumento de la mejor agudeza visual corregida en las distintas visitas y que este es independiente del sexo de los pacientes pero no de su edad. La evolución de la agudeza visual es estadísticamente diferente en pacientes con setenta y cinco años o menos que en aquellos con más de 75 años (fig. 10).

\section{DISCUSIÓN}

\section{Cuestionario SF-36}

Las dimensiones exploradas reflejan los siguientes datos

Funcionalidad física: Esta mejoría significativa encontrada se refleja en actividades de la vida diaria como pasear, subir escaleras, agacharse, bañarse y vestirse; una mejora en este aspecto podría indicar una mayor seguridad de los enfermos a la hora

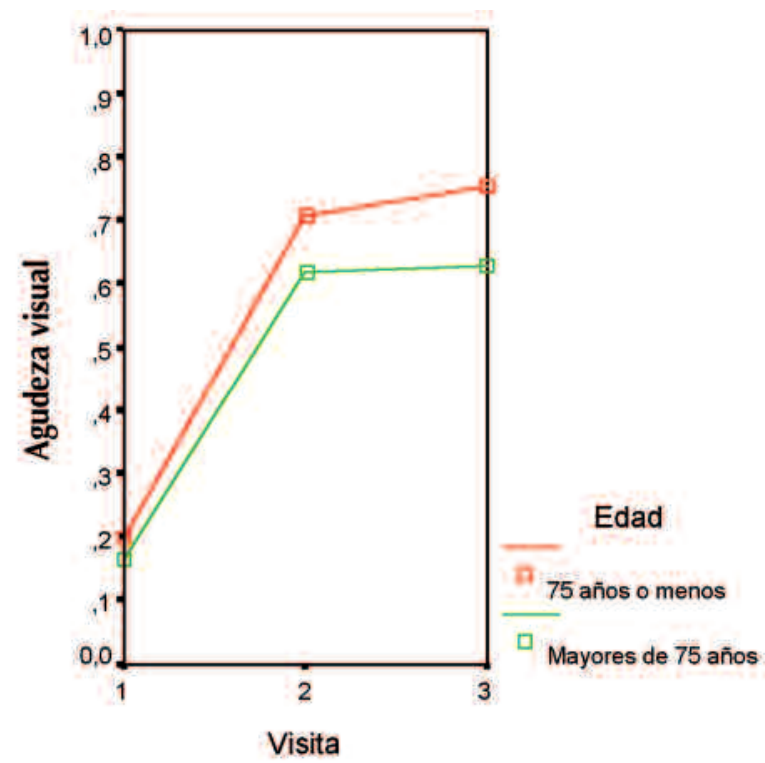

Fig. 10: Evolución de la agudeza visual de los pacientes intervenidos de catarata en las tres consultas realizadas separados en pacientes con 75 años o menos y mayores de 75 años. de afrontar sus tareas habituales. La función visual es la capacidad para realizar actividades que dependen de la visión, esto incluye las tareas domésticas, el cuidado personal y la movilidad.

Limitación de roles debido a problemas de salud físicos: La mejora en este aspecto concuerda con lo anterior, disminuyéndose las limitaciones experimentadas previas a la cirugía. Mejora la salud física y por tanto los problemas para realizar las actividades laborales o cotidianas. La intervención de cataratas puede evitar la pérdida de autonomía y retrasar las situaciones de dependencia.

Funcionalidad social: La mejora postquirúrgica podría indicar que aumenta la capacidad de los enfermos para tener una mayor autonomía, pudiendo realizar actividades sociales con más frecuencia. Mide la actividad social de los participantes en función del número de contactos y actividades y en base a la frecuencia de las mismas. Mejoran las relaciones y actividades sociales, en concordancia con la mejoría en las funciones físicas y emocionales.

Percepción de la propia salud: Mejora significativamente. Mide el sentimiento de la propia salud, la creencia de estar o no sano y de empeorar en un futuro.

Vitalidad, energía o fatiga: El aumento en esta dimensión no ha sido significativo pudiendo encontrarse una mayor sensibilidad emocional por parte de estos pacientes de elevada edad, más fácilmente influenciables .Esta dimensión se basa en la medida de cuatro aspectos que presentan una validez empírica y un correcto balance entre lo favorable y desfavorable de las respuestas dadas. La sensibilidad de la escala en el impacto de la enfermedad y tratamiento ha sido demostrada en ensayos clínicos (12-15).

Dolor corporal: Hay una mejoría significativa. Refleja el sentimiento de dolor y las limitaciones debidas al mismo. Hace referencia a la frecuencia y al tiempo que interfiere en las actividades diarias. La cirugía de catarata supone poca agresión y una recuperación rápida que se refleja en este cuestionario.

Rol emocional (disfunción social por problemas emocionales): La mejoría de la función social en el trabajo y en las actividades diarias debida a problemas emocionales observada no es significativa, sin embargo, la mayor función social obtenida hace que probablemente estos pacientes tengan menos tiempo para acordarse de otros problemas.

Salud actual comparada con la de hace un mes: Hay una mejoría significativa entre la primera y tercera consulta. 
Salud mental: Refleja un empeoramiento significativo probablemente porque la intervención influya de manera decisiva en aquellos pacientes con problemas emocionales de base. Representa las cuatro dimensiones mayores de la salud mental: ansiedad, depresión, pérdida del autocontrol y bienestar psicológico. Completa el rango desde el nerviosismo y la depresión, hasta la tranquilidad, felicidad y calma. Hay diversos estudios sobre la influencia de la cirugía de catarata en pacientes con disfunción cognitiva (16).

Se considera que cuantos más parámetros o dimensiones del SF-36 presenten una mejoría postquirúrgica, más probable será que la cirugía de catarata sea de utilidad clínica y suponga una mejora funcional para el paciente.

\section{Agudeza visual}

La agudeza visual de los pacientes intervenidos aumenta claramente tras la cirugía, siendo en la última visita aproximadamente cuatro veces mayor que la presente antes de la cirugía, y la mejoría es mayor en pacientes de menor edad. Esto puede no significar una mejor función visual ni una mayor capacidad para la vida independiente. Otros estudios coinciden concluyendo que la independencia para realizar actividades básicas y la edad inferior a 75 años, son variables que intervienen favorablemente en el resultado (17). La calidad de vida en sus aspectos más relacionados con la mejora visual presentan una evolución positiva.

\section{Cuestionario SF-36 y agudeza visual}

Actualmente cada vez es mayor el interés puesto en valorar la función visual y la calidad de vida relacionada con la salud y la visión. La relación entre ambas es evidente y queda reflejada con los resultados presentados. Se pueden considerar los resultados obtenidos como un INDICADOR DE CALIDAD ASISTENCIAL, mediante el cual se podría comparar, basándonos en los mismos criterios de inclusión de pacientes, la variación de la calidad de vida de los pacientes intervenidos en distintos hospitales. La valoración de los resultados de la cirugía de catarata sólo con indicadores clínicos puede infraestimar los beneficios globales, particularmente en pacientes con pobre resultado visual. Hay estu- dios que afirman que la mayor ganancia en función visual y en calidad de vida relacionada con la salud y la visión aparecen en el subgrupo de paciente con pobre resultado visual. La utilidad de estas medidas de la calidad de vida o similares en la valoración rutinaria de los resultados de la cirugía de catarata, necesita una valoración más extensa y profunda por la necesidad que conlleva de una correcta medición e interpretación de los resultados obtenidos y su interrelación con factores clínicos y demográficos. En los últimos diez años los ensayos clínicos en Oftalmología han ido incorporando progresivamente la percepción del paciente de su estado de salud general y los resultados de instrumentos de medida de calidad de vida relacionada con la visión (18).

La complejidad de la visión hace que la elaboración de un instrumento de medición ideal sea difícil. Pero, es fundamental que mientras se desarrolle, los facultativos prueben y evalúen los cuestionarios ya validados para mejorarlos y obtener mayor información de sus resultados $(19,20)$.

La cirugía de catarata es efectiva mejorando la visión y las funciones de la calidad de vida. Hay estudios que ya utilizan la medición de la calidad de vida como instrumento de decisión terapéutica (21). Nos parece importante destacar una sensibilidad cada vez mayor por parte de los médicos para incluir la mejora de calidad de vida de los enfermos tanto en los algoritmos diagnóstico terapéuticos cómo en el éxito de los resultados. Nuestro trabajo pretende caminar hacia la asistencia integral de los pacientes, buscando una mejora no sólo de aspectos técnicos, sino que además dicha mejora quede reflejada en la mejora de calidad de vida de los enfermos.

Podemos concluir:

La facoemulsificación con implante de lente intraocular es un método efectivo y seguro para mejorar la Calidad de vida, sobre todo en los aspectos físicos.

La disminución de la limitación de roles debido a problemas de salud físicos tras la cirugía es concordante con la mejora física, mejorando la funcionalidad de los enfermos en los aspectos cotidianos de su vida.

La mejora en las relaciones sociales confirma la importancia de la medición de aspectos de calidad de vida en los resultados de cualquier intervención médica.

La agudeza visual aumenta claramente tras la cirugía, siendo en la última visita aproximadamente cuatro veces mayor que la presente antes de la cirugía. 
Los cuestionarios genéricos de calidad de vida como el SF-36 se pueden utilizar como medida de resultado de la cirugía de catarata.

\section{BIBLIOGRAFÍA}

1. Kahn HA, Leibowitz HM, Ganley JP, Kini MM, Colton T, Nickerson RS, et al. The Framinghan Eye Study. I. Outline and major prevalence findings. Am J Epidemiol 1977; 106: 17-32.

2. Klein BE, Klein R, Linton KL. Prevalence of age-related lens opacities in a population. The Beaver Dam Eye Study. Ophthalmology 1992; 99: 546-552.

3. Steinberg EP, Javitt JC, Sharkey PD, Zuckerman A, Legro $M W$, Anderson $G F$, et al. The content and cost of cataract surgery. Arch Ophthalmol 1993; 111: 1041-1049.

4. Muldoon MF, Barger SD, Flory J, Manuck SB. What are quality of life measurements measuring? BMJ 1998; 316: 542-545.

5. Sanders C, Egger M, Donovan J, Tallon D, Frankel S. Reporting quality of life in randomised controlled trials: bibliographic study. BMJ 1998; 317: 1191-1194.

6. de Boer MR, Moll AC, de Vet HC, Terwee CB, Völker-Dieben HJ, van Rens GH. Psychometric properties of visionrelated quality of life questionnaires: a systematic review. Ophthalmic Physiol Opt 2004; 24: 257-273.

7. Lee JE, Fos PJ, Zuniga MA, Kastl PR, Sung HJ. Assesing health-related quality of life in cataract patients: the relationship between utility and health-related quality of life measurement. Qual Life Res 2000; 9: 1127-1135.

8. Desai P, Reidy A, Minassain DC, Vafidis G, Bolger J. Gains from cataract surgery: visual function and quality of life. Br J Ophthalmol 1996; 80: 868-873.

9. Lanksy D, Butler JB, Waller FT. Using health status measures in the hospital setting: from acute care to "outcomes management». Med Care 1992; 30: MS57-MS73.

10. Ware JE Jr, Sherbourne CD. The MOS 36-item short-form health survey (SF-36). I. Conceptual framework and item selection. Medical Care 1992; 30: 473-483.
11. Cabezas-León M, Gracia-San Román J, García-Caballero J, Morente-Matas P. Calidad de vida en pacientes intervenidos de catarata. Arch Soc Esp Oftalmol 2005; 80: 449-456.

12. Fowler FJ Jr, Wennberg JE, Timothy RP, Barry MJ, Mulley AG Jr, Hanley D. Symptom status and quality of life following prostatectomy. JAMA 1988; 259: 30183022.

13. Berwick DM, Murphy JM, Goldman PA, Ware JE Jr, Barsky AJ, Weinstein MC. Performance of a five-item mental health screening test. Med Care 1991; 29: 169176.

14. Croog SH, Levine S, Testa MA, Brown B, Bulpitt CJ, Jenkins $C D$, et al. The effects of antihypertensive therapy on quality of life. N Engl J Med 1986; 314: 1657-1664.

15. Wu AW, Rubin HR, Mathews WC, Ware JE Jr, Brysk LT, Hardy WD, et al. A health status questionnaire using 30 items from the Medical Outcomes Study: Preliminary validation in persons with early HIV infection. Med Care 1991; 29: 786-798.

16. Brenner MH, Curbow B, Javitt JC, Legro MW, Sommer A. Vision change and quality of life in the elderly. Response to cataract surgery and treatment of other chronic ocular conditions. Arch Opthalmol 1993; 111: 680-685.

17. López-Torres Hidalgo J, López, Verdejo MA, Otero Puime A, Belmonte Useros M, López Verdejo J, Montoro Durán J. Repercusión de la intervención de cataratas en la capacidad funcional del anciano. Arch Soc Esp Oftalmol 2004; 79: 221-228.

18. Godlee F. The Cochrane collaboration. BMJ 1994; 309: 969-970.

19. D'Ambrosio FA Jr. Assessing disability in the patient with cataracts. Curr Opin Ophthalmol 1999; 10: 42-45.

20. Globe DR, Levin MS, Chang TS, Mackenzie PJ, Azen S. Validity of the SF-12 quality of life instrument in patients with retinal diseases. Ophthalmology 2002; 109: 17931798.

21. Janz NK, Wren PA, Lichter PR, Musch DC, Gillespie BW, Guire KE. Quality of life in newly diagnosed glaucoma patients: The Collaborative Initial Glaucoma Treatment Study. Ophthalmology 2001; 108: 887-898. 